\title{
EFECTO DE FACTORES ECONÓMICO - SOCIALES Y MODALIDAD DE INGRESO EN EL RENDIMIENTO ACADÉMICO: FACULTAD DE ECONOMÍA 2005-2010
}

\author{
IMPACT OF ECONOMIC FACTORS - SOCIAL AND METHOD OF ENTRY \\ ACADEMIC ACHIEVEMENT : 2005-2010 FACULTY OF ECONOMICS
}

\author{
Miguel Huaringa Sánchez, María E. Aliaga Guerra
}

\section{RESUMEN}

El presente trabajo de investigación, se formuló con el propósito de determinar el efecto de los factores socioeconómicos y de la modalidad de ingreso en el rendimiento académico de los estudiantes de la facultad de Economía, evaluada en dos cohortes la del 2005 y la del 2008, teniendo en consideración que la formación de nuestros estudiantes, no solo se da como resultado de lo que acontece en las aulas, sino que también influyen otros factores relacionados a los aspectos socioeconómicos, por lo que es de primordial importancia conocer el perfil de nuestros ingresantes y contrastarlo con el perfil que se requiere. Para el efecto se utilizó un diseño ex post facto, tomando información de los expedientes académicos de los estudiantes seleccionados en la muestra proporcionada por la Oficina de Registros Académicos de la Facultad y de los registros de sus respectivas fichas socioeconómicas. Se hizo dos regresiones, una para el rendimiento académico en nota en el primer semestre de estudio, y la otra con el promedio ponderado del último semestre cursado en la fecha de corte del análisis, encontrando diferentes resultados en cada caso. Se encontró que la variable modalidad de ingreso no influye en el rendimiento académico de los estudiantes de la facultad de Economía. Cuando los estudiantes están en la etapa de adaptación a la vida universitaria, las variables que influyen en su rendimiento académico son: la edad de ingreso y el turno de estudios. Una vez superada esta etapa de adaptación, surgen otras variables para explicar el rendimiento académico de los estudiantes de la facultad de Economía, ellas son: el tipo de colegio donde cursaron los estudios secundarios y el lugar de origen

\section{ABSTRACT}

This research work was formulated for the purpose of determining the effect of the factors socioeconomic and mode of entry into the academic performance of students of the faculty Economy evaluated in two cohorts of 2005 and 2008, taking into consideration that the formation of our students, not only is a result of what happens in the classroom, but also influence others factors related to socioeconomic aspects, so it is of paramount importance to know the profile our entrants and contrast it with the profile required. For this purpose we used an ex post facto design, taking information from the transcripts of the selected in the sample provided by the Office of Academic Records of the College Students and records of their socioeconomic chips. Two regressions were made, one for performance academic in note in the first semester of study, and the other with the weighted average last semester studied at the cutoff date of the analysis, finding different results in each case. It was found that the variable input mode does not affect the academic performance of students of the faculty Economy. When students are in the process of adaptation to college life, the variables that influence their academic performance are: the age of entry and shift studies. Once this stage adaptation arise other variables to explain the academic performance of students of the faculty Economy, they are the type of school where coursed secondary education and the place of origin. 


\section{INTRODUCCIÓN}

En la Universidad Nacional del Centro del Perú, como reflejo de las tendencias que exige un mundo globalizado, se ha afianzado el camino hacia el modelo curricular por competencias, en el cual se toma un especial empeño en el desarrollo de capacidades profesionales de los egresados, lo que implica hablar de un perfil del egresado y hacia el van encaminados los esfuerzos de la institución por mejorar las competencias de los nuevos profesionales formados en nuestras aulas universitarias; en consecuencia, los principales estudios y las investigaciones se centran en las fortalezas de la institución tales como: capacidad docente (actualización y capacitación en docencia universitaria); infraestructura física; biblioteca especializada; laboratorios; centros de aplicación; actualización de los planes de estudios; convenios para prácticas pre profesionales; programas de movilidad estudiantil, etc.

Sin embargo, como manifiesta Idrovo (2008), es necesario además, identificar el perfil del ingresante, y más aún, conocer cuál es el efecto de los diferentes aspectos económicos y sociales, tales como: familia, colegio y lugar de procedencia en el rendimiento académico de los estudiantes de la UNCP., para establecer políticas educativas que deban ser consideradas en el Proyecto Educativo Institucional. Por otro lado, en muchas facultades no se tiene bien en claro el perfil del ingresante, y lo que es más crítico, el Examen de Admisión que sirve para clasificar a los postulantes más aptos para seguir estudios universitarios, muchas veces no toma en cuenta los requerimientos del perfil del ingresante, resultado del cual, los niveles de deserción y repitencia estudiantil son significativos. El presente estudio, plantea identificar el efecto de las variables económico-sociales (con los que ingresa el estudiante a la facultad de Economía) en su rendimiento académico y observar, a lo largo de su vida estudiantil como ese efecto se va desvaneciendo paulatinamente a medida que el estudiante se afianza en la carrera, con el avance de sus estudios. El objetivo general del presente trabajo es el de determinar cuál fue el efecto de la modalidad de ingreso y los aspectos económico-sociales, sobre el rendimiento académico de los estudiantes de la Facultad de Economía UNCP en el periodo 2005-2010.

\section{Los objetivos específicos son:}

- Analizar el efecto de la modalidad de ingreso en el rendimiento académico de los estudiantes de la facultad de Economía UNCP 20005-2010.

- Analizar el efecto de los factores económico-sociales en el rendimiento académico de los estudiantes de la facultad de Economía UNCP 20005-2010.

\section{MATERIAL Y MÉTODOS}

La investigación es de tipo explicativa, pues se indagó si las características familiares y la modalidad de ingreso, explicaban el rendimiento académico de los estudiantes de la facultad de Economía UNCP.

La población estuvo constituida por los estudiantes de la Facultad de Economía de la UNCP

La muestra fue conformada por los estudiantes que lograron su ingreso en el año 2005 y en el año 2008 contando de esta manera con dos cohortes en las cuales efectuar el análisis, la primera constituía una muestra de estudiantes con el Plan 1995, mientras que la segunda era una muestra del Plan 2008.

La sistematización de los resultados se efectuó con el uso de la hoja de cálculo Excel 2007, del software estadístico SPSS y del software econométrico Eviews.

El diseño de investigación es un diseño ex post facto, pues se analizó un fenómeno que ya ocurrió. (performance académica de los estudiantes del facultad de economía en el periodo 2005-2010; 2008-2012 ).

\section{VARIABLE E INDICADORES}

Variable dependiente: Rendimiento académico cuyos indicadores son: Promedio ponderado acumulado en el sistema vigesimal; situación del estudiante(Normal, pagante permanente, pagante transitorio, suspendido); y condición del estudiante (alto rendimiento, invicto, regular, repitente).

Variables independientes: modalidad de ingreso (Examen de Admisión Regular, por la modalidad de estudios en el Centro Pre Universitario Primera Selección, primeros puestos, traslado externo y traslado interno.

Factores económicos: Ingreso familiar, dependencia económica del estudiante, gastos en educación.

Factores sociales: sexo de los estudiantes, grado de estudios de los padres de familia, colegio de procedencia, lugar de origen. 


\section{RESULTADOS}

\section{Analisis descriptivo}

Considerando el análisis de dos cohortes, se muestran los principales resultados descriptivos encontrados.

Se consideró el turno de estudios (mañanas y tardes) del primer y segundo semestre de facultad.

Cuadro $\mathrm{N}^{\circ} 01$. Estudiantes por cohorte y por turno de estudio

\begin{tabular}{|c|c|c|c|}
\hline \multirow{2}{*}{ COHORTE } & \multicolumn{2}{|c|}{ TURNO DE ESTUDIOS } & \multirow{2}{*}{ Total } \\
\cline { 2 - 3 } & MAÑANAS & TARDES & \\
\hline 2005 & 44 & 0 & 44 \\
\hline 2008 & 25 & 22 & 47 \\
\hline Total & 69 & 22 & 91 \\
\hline
\end{tabular}

Se hizo el análisis de los estudiantes por cohorte, en función del tipo de centro educativo secundario de procedencia (público y privado) de los estudiantes de la facultad de Economía. De igual manera en función del tipo de ocupación del padre y de la madre, cuyos resultados se muestran en los cuadros 3 y 4.

\section{Cuadro $\mathrm{N}^{\circ}$ 02. Estudiantes por cohorte y por tipo} de colegio

\begin{tabular}{|c|c|c|c|}
\hline \multirow{2}{*}{ COHORTE } & \multicolumn{2}{|c|}{ COLEGIO } & \multirow{2}{*}{ Total } \\
\cline { 2 - 3 } & PUBLICO & PRIVADO & \\
\hline $\mathbf{2 0 0 5}$ & 33 & 11 & 44 \\
\hline $\mathbf{2 0 0 8}$ & 38 & 9 & 47 \\
\hline Total & 71 & 20 & 91 \\
\hline
\end{tabular}

Cuadro $\mathrm{N}^{\circ} 03$. Estudiantes por cohorte y por ocupación del padre

\begin{tabular}{|c|c|c|c|}
\hline \multirow{2}{*}{ OCUPACION DEL PADRE } & \multicolumn{2}{|c|}{ COHORTE } & \multirow{2}{*}{ Total } \\
\hline & 2005 & 2008 & \\
\hline Fallecio & 3 & 0 & 3 \\
\hline Obrero & 4 & 4 & 8 \\
\hline Agricultor & 4 & 5 & 9 \\
\hline Jubilado & 1 & 0 & 1 \\
\hline Trabajador dependiente & 8 & 3 & 11 \\
\hline Profesional dependiente & 13 & 11 & 24 \\
\hline Trabajador independiente & 8 & 20 & 28 \\
\hline Profesional independiente & 3 & 4 & 7 \\
\hline Total & 44 & 47 & 91 \\
\hline
\end{tabular}

Cuadro $\mathrm{N}^{\circ} 04$. Estudiantes por cohorte y por ocupación de la madre

\begin{tabular}{|c|c|c|c|}
\hline \multirow{2}{*}{ OCUPACION DE LA MADRE } & \multicolumn{2}{|c|}{ COHORTE } & \multirow{2}{*}{ Total } \\
\hline & 2005 & 2008 & \\
\hline Ama de casa & 25 & 24 & 49 \\
\hline Agricultor & 2 & 1 & 3 \\
\hline Trabajadora dependiente & 3 & 0 & 3 \\
\hline Profesional dependiente & 7 & 6 & 13 \\
\hline Trabajador independiente & 7 & 16 & 23 \\
\hline Total & 44 & 47 & 91 \\
\hline
\end{tabular}

También se efectuó el análisis de la distribución de los estudiantes por cohortes y en función de la modalidad de ingreso a la UNCP y en función de la edad al momento del ingreso, los resultados se muestran en los cuadros 5 y 6 .

\section{Cuadro $\mathrm{N}^{\circ} 05$. Estudiantes por cohorte y por tipo} de ingreso

\begin{tabular}{|c|c|c|c|c|c|c|}
\hline \multirow[b]{2}{*}{ COHORTE } & \multicolumn{5}{|c|}{ TIPO DE INGRESO } & \multirow[b]{2}{*}{ Tota } \\
\hline & 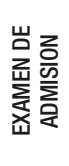 & 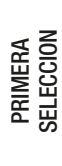 & 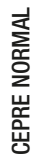 & 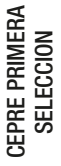 & 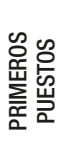 & \\
\hline 2005 & 21 & 12 & 6 & 3 & 2 & 44 \\
\hline 2008 & 39 & 0 & 6 & 0 & 2 & 47 \\
\hline Total & 60 & 12 & 12 & 3 & 4 & 91 \\
\hline
\end{tabular}

Cuadro $\mathrm{N}^{\circ} 06$. Estudiantes por cohorte y por edad en la fecha de ingreso

\begin{tabular}{|c|c|c|c|}
\hline \multirow{2}{*}{$\begin{array}{c}\text { EDAD EN EL } \\
\text { INGRES0 }\end{array}$} & \multicolumn{2}{|c|}{ COHORTE } & \multirow{2}{*}{ Total } \\
\cline { 2 - 3 } & 2005 & 2008 & 11 \\
\hline 16 & 1 & 10 & 30 \\
\hline 17 & 16 & 14 & 25 \\
\hline 18 & 12 & 13 & 11 \\
\hline 19 & 5 & 6 & 7 \\
\hline 20 & 5 & 2 & 3 \\
\hline 21 & 2 & 1 & 2 \\
\hline 22 & 2 & 0 & 1 \\
\hline 25 & 0 & 1 & 1 \\
\hline 26 & 1 & 0 & 91 \\
\hline Total & 44 & 47 & \\
\hline
\end{tabular}


Los estudiantes fueron distribuidos por cohorte y lugar de origen, encontrando que una mayor parte de ellos proceden del Valle del Mantaro, que une a las provincias de Huancayo, Concepción, Chupaca y Jauja.

\section{Cuadro $\mathrm{N}^{\circ}$ 07. Estudiantes por cohorte y por lugar} de origen

\begin{tabular}{|c|c|c|c|c|c|c|c|}
\hline \multirow[b]{2}{*}{ COHORTE } & \multicolumn{6}{|c|}{ LUGAR DE ORIGEN } & \multirow[b]{2}{*}{ Total } \\
\hline & 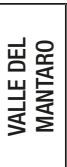 & 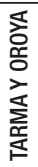 & 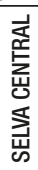 & 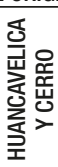 & 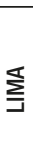 & $\begin{array}{l}\text { ஜ } \\
\text { ơ } \\
\end{array}$ & \\
\hline 2005 & 29 & 2 & 4 & 6 & 3 & 0 & 44 \\
\hline 2008 & 35 & 2 & 2 & 5 & 2 & 1 & 47 \\
\hline Total & 64 & 4 & 6 & 11 & 5 & 1 & 91 \\
\hline
\end{tabular}

Cuadro $\mathrm{N}^{\circ} 08$. Estudiantes por cohorte y por número de semestres retrazados

\begin{tabular}{|c|c|c|c|}
\hline \multirow{2}{*}{$\begin{array}{c}\text { RETRAZO EN SE- } \\
\text { MESTRE }\end{array}$} & \multicolumn{2}{|c|}{ COHORTE } & \multirow{2}{*}{ Total } \\
\cline { 2 - 3 } & $\mathbf{2 0 0 5}$ & $\mathbf{2 0 0 8}$ & \\
\hline 0 & 6 & 11 & 17 \\
\hline 1 & 8 & 4 & 12 \\
\hline 2 & 3 & 10 & 13 \\
\hline 3 & 8 & 7 & 15 \\
\hline 4 & 3 & 3 & 6 \\
\hline 5 & 3 & 4 & 7 \\
\hline 6 & 1 & 2 & 3 \\
\hline 7 & 6 & 4 & 10 \\
\hline 8 & 3 & 2 & 5 \\
\hline 9 & 3 & 0 & 3 \\
\hline Total & 44 & 47 & 91 \\
\hline
\end{tabular}

Cuadro $\mathrm{N}^{\circ} 09$. Estudiantes por cohorte y por su origen en el Valle del Mantaro

\begin{tabular}{|c|c|c|c|}
\hline \multirow{2}{*}{ COHORTE } & \multicolumn{2}{|c|}{ DICOTOMICA VALLE } & \multirow{2}{*}{ Total } \\
\cline { 2 - 3 } & OTR0 & V. del M. & \\
\hline 2005 & 15 & 29 & 44 \\
\hline 2008 & 12 & 35 & 47 \\
\hline Total & $\mathbf{2 7}$ & $\mathbf{6 4}$ & $\mathbf{9 1}$ \\
\hline
\end{tabular}

\section{PRUEBAS DE HIPOTESIS}

En primer lugar se hizo un análisis de correlación entre el promedio ponderado del primer semestre y el promedio ponderado del último semestre cursado, encontrando que existe una relación positiva entre ambas variables $(r=0,58)$, lo cual estaría indicando que la tendencia en los calificativos de nuestros estudiantes, está influenciada por su rendimiento en los primeros semestres, donde se afianzan los conocimientos básicos para la ciencia económica.

Gráfico $\mathrm{N}^{\circ} 01$. Relación entre los promedios ponderados del I semestre de facultad y el último semestre cursado

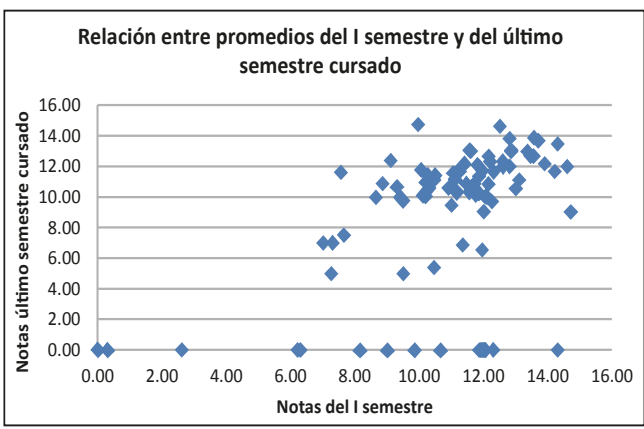

\section{MODELO DE REGRESION LINEAL}

Desarrollándose una investigación explicativa, para el rendimiento académico del primer semestre de estudio, se encontró que las variables socioeconómicas más significativas fueron, cohorte, edad de ingreso, retrazo (repitencia) y turno de estudios. El modelo tiene una significación estadística global, y su bondad de ajuste es de 40,98\% (R cuadrado ajustado). Los resultados, se encuentran en el cuadro $\mathrm{N}^{\circ} 10$

\section{Cuadro $\mathrm{N}^{\circ} 10$. Variables que explican el Rendimiento Académico en el I Semestre de Estudios}

\begin{tabular}{|c|c|c|c|c|}
\hline \multicolumn{3}{|c|}{ Dependent Variable: NOTA1 } & & \\
\hline \multicolumn{3}{|c|}{ Method: Least Squares } & & \\
\hline \multicolumn{2}{|c|}{ Date: 09/18/12 Time: 23:51 } & & & \\
\hline \multicolumn{2}{|l|}{ Sample: 191} & & & \\
\hline \multicolumn{5}{|c|}{ Included observations: 91} \\
\hline Variable & Coefficient & Std. Error & t-Statistic & Prob. \\
\hline C & 20.43579 & 3.257594 & 6.273277 & 0.0000 \\
\hline COHORTE & -2.452146 & 0.674723 & -3.634301 & 0.0005 \\
\hline EDADING & -0.330359 & 0.160252 & -2.061500 & 0.0423 \\
\hline RETRAZO & -0.771921 & 0.104222 & -7.406486 & 0.0000 \\
\hline
\end{tabular}




\begin{tabular}{|c|c|c|c|}
\hline TURNO & 1.738316 & $0.774039 \quad 2.245772$ & 0.0273 \\
\hline R-squared & 0.436089 & Mean dependent var & 10.39176 \\
\hline Adjusted R-squared & 0.409860 & S.D. dependent var & 3.427489 \\
\hline S.E. of regression & 2.633016 & Akaike info criterion & 4.827515 \\
\hline Sum squared resid & 596.2185 & Schwarz criterion & 4.965474 \\
\hline Log likelihood & -214.6519 & F-statistic & 16.62657 \\
\hline Durbin-Watson stat & 1.373105 & Prob(F-statistic) & 0.000000 \\
\hline
\end{tabular}

\section{Cuadro $\mathrm{N}^{\circ}$ 11. Variables que explican el Rendimiento Académico en el Último Semestre cursado}

\begin{tabular}{|c|c|c|c|c|}
\hline \multicolumn{3}{|c|}{ Dependent Variable: NOTA2 } & & \\
\hline \multicolumn{3}{|c|}{ Method: Least Squares } & & \\
\hline \multicolumn{2}{|c|}{ Date: 09/19/12 Time: 00:06 } & & & \\
\hline \multicolumn{2}{|l|}{ Sample: 191} & & & \\
\hline \multicolumn{5}{|c|}{ Included observations: 91} \\
\hline Variable & Coefficient & Std. Error & t-Statistic & Prob. \\
\hline C & 11.55141 & 1.642190 & 7.034154 & 0.0000 \\
\hline COLE2 & -1.623612 & 0.768153 & -2.113657 & 0.0374 \\
\hline NOTA1 & 0.302577 & 0.108811 & 2.780763 & 0.0067 \\
\hline ORIGEN2 & -1.210914 & 0.695942 & -1.739965 & 0.0854 \\
\hline RETRAZO & -1.246450 & 0.136600 & -9.124848 & 0.0000 \\
\hline R-squared & 0.671506 & \multicolumn{2}{|c|}{ Mean dependent var } & 8.509231 \\
\hline Adjusted R-squared & 0.656227 & \multicolumn{2}{|c|}{ S.D. dependent var } & 4.905294 \\
\hline S.E. of regression & 2.876081 & \multicolumn{2}{|c|}{ Akaike info criterion } & 5.004112 \\
\hline Sum squared resid & 711.3783 & \multicolumn{2}{|c|}{ Schwarz criterion } & 5.142071 \\
\hline Log likelihood & -222.6871 & \multicolumn{2}{|c|}{ F-statistic } & 43.95011 \\
\hline Durbin-Watson stat & 2.092378 & \multicolumn{2}{|c|}{ Prob(F-statistic) } & 0.000000 \\
\hline
\end{tabular}

De igual manera se especificó un modelo considerando el promedio obtenido en el último semestre estudiado al culminar el análisis, encontrando que las variables significativas que explican el rendimiento académico de los estudiantes de la facultad de economía son: Tipo de colegio, el promedio ponderado obtenido en el primer semestre de facultad, si su procedencia es el Valle del Mantaro, y el número de semestres repetidos.

\section{DISCUSIÓN}

De acuerdo a los resultados obtenidos en la primera regresión, se puede encontrar que el rendimiento académico del I semestre de estudios representado por su promedio ponderado, es explicado por el Plan de Estudios (cohorte) que nos está indicando que el rendimiento académico de los estudiantes con el nuevo plan de estudios (cohorte 2008), tienen un calificativo menor de 2,45. La edad en el momento de ingreso influye en el promedio ponderado obtenido en el primer semestre, así como el retrazo en número de semestres desaprobados y el turno de estudio, pues los estudiantes del turno de mañanas presentan un mejor desempeño (una diferencia de 1,73 puntos).

En la segunda regresión, se encontró que el promedio ponderado de los estudiantes en el último semestre cursado, es explicado por el tipo de colegio donde estudiaron la secundaria, teniendo un mayor desempeño quiénes estudiaron en colegios privados. De igual manera, la performance académica obtenida en el primer semestre ayuda a explicar el rendimiento académico obtenido en el semestre de salida; así como el origen de los estudiantes (aunque no muy significativa), y el número de semestres que se retrazaron frente a los invictos.

Las variables socioeconómicas como: edad en el ingreso, ocupación de los padres y madres, Plan de estudios, turno de estudios, y modalidad de ingreso, no explican significativamente el rendimiento académico de los alumnos de semestres superiores en la facultad de Economía de la UNCP.

\section{CONCLUSIONES}

1. La modalidad de ingreso, no tiene efecto en el rendimiento académico de los estudiantes de la facultad de economía.

2. Las variables socioeconómicas que explican el comportamiento del rendimiento académico de los estudiantes de la facultad de economía son: la edad de ingreso y el turno de estudios, en el primer semestre de facultad.

3. una vez superada esa primera etapa de adecuación a la vida estudiantil, surgen otras variables socioeconómicas para explicar el comportamiento académico de las estudiantes, tales como: el tipo de colegio donde cursaron los estudios secundarios y el lugar de origen. 


\section{REFERENCIAS BIBLIOGRÁFICAS}

1. CANALI Lidia (2007). Fortalezas y debilidades de la docencia universitaria. II encuentro nacional de docentes universitarios católicos. Argentina.

2. CANO GARCÍA E. (2000). ¿Qué significa evaluar?. Revista Innovación educativa. Universidad de Málaga.

3. FERNANDEZ Adrián, Carolina FIRPO y Marcelo PERERA (2000). Proyección de la matrícula universitaria periodo 2000-2030. Documento de trabajo Universidad de la República. Uruguay.

4. HUARINGA Miguel et al (2005). La Evaluación para el Ingreso a la UNCP.

5. HUARINGA M. María Aliaga (2010). Efecto de los agentes educadores y modalidad de ingreso en el rendimiento académico de los estudiantes de la UNCP. Caso: Facultad de Economía.

6. IDROVO Byron. (2007) ¿Son las escuelas particulares subvencionadas mejores que las municipales?
Estimación de la ecuación de logro escolar para Chile. Tesis Magister. Universidad de Chile.

7. MOLNAR Gabriel: (2008). Concepto de evaluación aplicada.

8. PULIDO San Román (2003). Propuesta metodológica para le evaluación de la calidad docente e investigadora. Universidad Autónoma de Madrid.

9. QUEZADA ZEVALLOS Jenny (2004). Retos en la docencia universitaria del siglo XXI. UNIFE. Centro de Orientación Pedagógica.

10. VELEZ Eduardo, Ernesto SCHIEFELBEIN, Jorge VALENZUELA (1995). Factores que afectan el rendimiento académico. Revisión de Literatura América Latina y El Caribe.

11. ZABAL Antoni, Laia ARNAU (2008). Como aprender y enseñar competencias. Tercera Edición. Editorial GRAÓ. Barcelona. 